\title{
Sinergic anti-GVHD and anti-tumour effect Of Ruxolitinib in the post allo transplant of myeloid diseases?
}

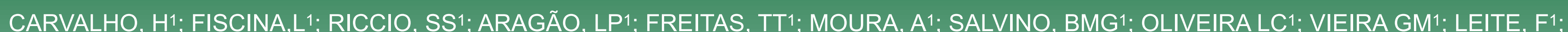
SALVINO MA1

1 Hospital São Rafael, Salvador, Bahia, Brazil

\section{Introduction:}

Acute Myeloid Leukemia (AML) represents a group of heterogenous diseases, extreme aggressive, with high rates of relapse and high mortality The bone marrow transplantation (BMT) is one the best treatment for this kind of malignant disease, with potential of cure. However, BMT present with high rates of mortality related to the treatment, Graft versus Hos (GVHD) it's the disease (GVHD) it's the principal complicatons. When compart others hematologic diseases, AML is fare behind in new therapies. In th last few years, with better understand in molecular biology of the diseases news approaches wherein comes translating in alternative treatment. Th JAK-STAT is a kinase is responsible by the differentiation of the hematopoietic cells and also is related the several processes inflammator cytokines. This regulatory kinase is some what mutated in variety myelo clignant diseases and also, it's nos that in GHVD, JAK-STAT is hig malignant diseases and also, it's notes that in GHVD, JAK-STAT is hig express in many pathways of signalling in the production of th inflammatory cytokines.

\section{Obiectives:}

This current study aim to demonstrate that JAK-STAT inhibition presents a satisfactory alternative to control both pathologies. It's describe 03 cases o patients with diagnosis of AML, submitted to allogenic BMT and

\section{Case 01:}

DSSM, 26 years, female, with AML with FLT3 positive in 2013, was submitted to allogenic BMT in first remission. However, in D+90 the disease progressed, then was submitted to second line therapy and a second BMT with the same donor, without prophylaxis to GVHD, that occurred without complication. Soon after, she developed with acute skin GVHD, refractory to corticosteroid and partial response to photoaferesis. About $\mathrm{D}+60$, it's was note tumor formation in sternun region, and further confirmed to be granulocytic sarcoma. Without disease in bone marrow, the patient was submitted to sorafenib, azacitidine and radiotherapy, with remission of the tumor. The patient hold the treatment well (Azacitidine was used for 03 years), without AML progression, however, during that period, presented with extensive skin chronic GHVD of associated with syndrome sicca. It's was used high doses of corticosteroids and photoaferesis (held total of 24 sessions), with partial response. It's proposed to used ruloxitinob to GVHD control. It was initiated in $15 \mathrm{mg}$

\section{Case: 02}

ACOO, 51 years, female, with diagnosis of AML in 2014, submitted the induction therapy with "7+3", presenting several infectious complications during the treatment. In remission, submitted to allogenic BMT in january 2015. Presents with acute skin GVHD with remission after corticosteroids. After $\mathrm{d}+100$, it was start maintenance with azacitidine, which used for 12 months. In $\mathrm{D}+120$, the patient developed extensive chronic GVHD, involving skin, lungs and muccosa. It was treated, agoressively, with corticosteroid, rituximab and imatinib, with pulmonary symptoms improvement, keeping a partial response to muccousa and skin, with worsened during the weaning of corticosteroid. In july 2017, started the use of ruxolitinib, $15 \mathrm{mg}$ / day with increase further to $30 \mathrm{mg} /$ day, presenting with stable GVHD, without worsening the lesions and enabled to weaning the corticosteroid. Patient follows the use, without adverse effects.
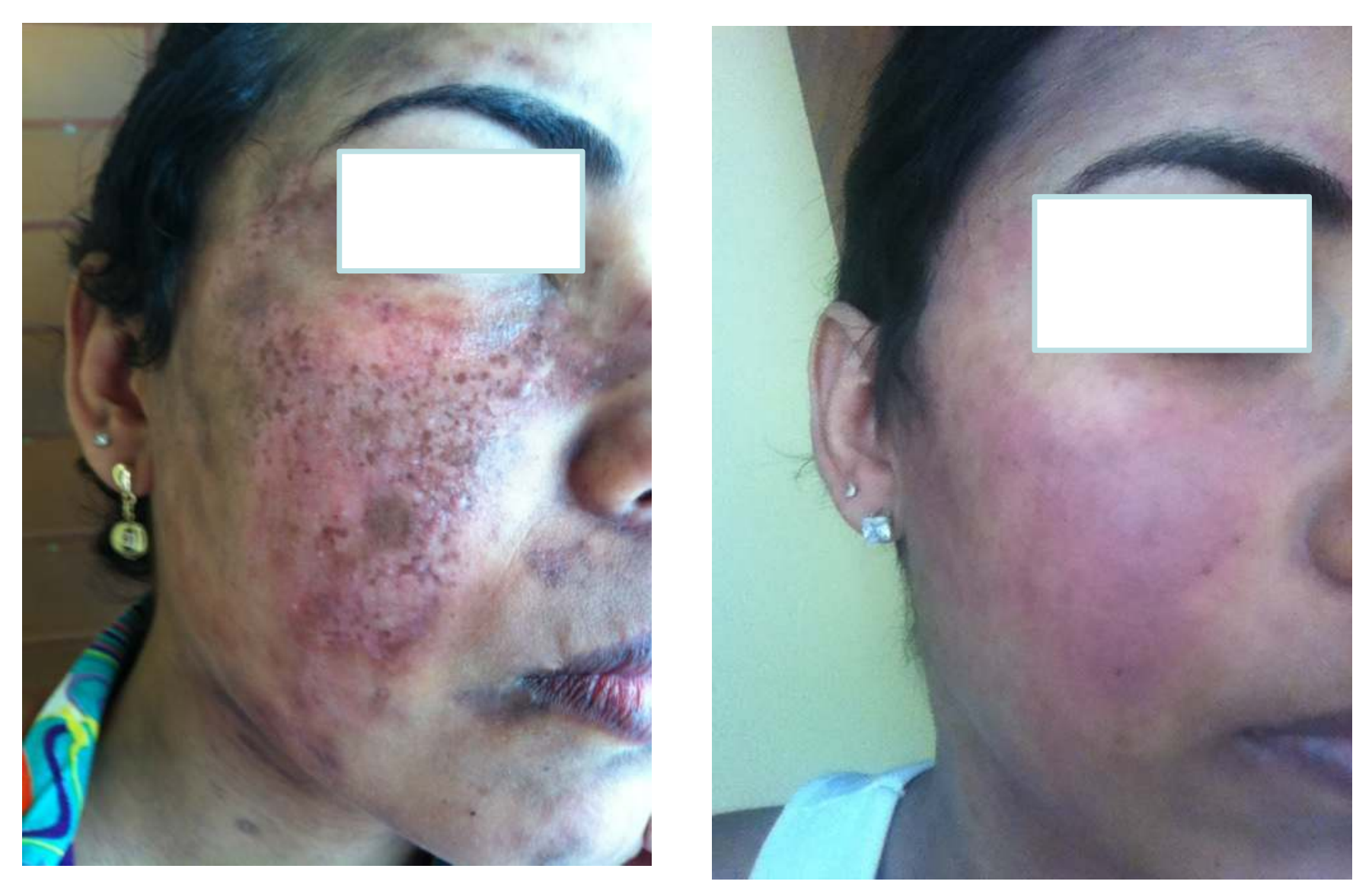

Figure 1. Before and After Ruxolitinib
EMSP 56 years, female with diagnosis AML FLT3 negative in january of 2017. Submitted to induction therapy with "7 + 3", which presents with various complication. Treated with 01 cycle of consolidation with high dose of cytarabine and followed allogenic BMT. Processed without complication, and in $\mathrm{D}+30$, was initiated maintenance therapy with azacitidine. In $\mathrm{D}+60$, patient presents with pancitopenia and futher investigation, it was confirmed progress of disease. Submitted to second line therapy and allogenic BMT with the same donor, without prophylaxis to GVHD. It was without major complication. About D+30, patient didn't show any signs of GVHD, and was proposed DLI infusion. After two sessions, the patient developed acute skin GVHD, with responded to corticosteroid. However, in the $d+100$, new skin lesions appeared, with characteristics of Chronic skin GVHD. In use of corticosteroid, patient presented with worsening of the lesions, further it was performed immunophenotyping, that MRD positive. Chosen by weaning complete of corticosteroid and started ruxolitinib. In the time, patient is the $d+150$, with stable skin GVHD, keeping MRD positive but without increase of the population.

\section{Conclusion:}

The present study describe the possibility of use the JAK-STAT inhibitor as alternative treatment for control GVHD, wherein was possible to withdrawal by complete of the corticosteroid and the maybe control the myeloid disease. However, more studies are needed, with larger number of patients to evaluate the effectiveness of this therapy in this kind of patient. 